\title{
e-interview
}

\section{Marianne C. Kastrup}

Marianne Kastrup is Head of the National Centre for Transcultural Psychiatry, Copenhagen. She trained at the Rigshospitalet University Clinic, Copenhagen. Her special interests include cultural psychiatry, psychiatric ethics, human rights and women's issues.

If you were not a psychiatrist, what would you do?

I would have chosen another profession dealing with marginalised people. This might have been the public health of minority groups or as a judge with a focus on human rights.

Do you feel stigmatised by your profession?

I have often felt that I had to justify the psychiatric profession, in particular the use of coercion and, to some extent, the use of antipsychotic medication.

\section{What are your interests outside} of work?

Human rights groups such as Amnesty International, taking part in women's movements of various kinds, cycling, travel to European cities, flee markets and secondhand designer clothes.

Who was your most influential trainer, and why?

My first trainer, Annalise Dupont, who supported my career, gave me lots of opportunities and had high expectations that I fulfil them. Also, Norman Sartorius who has greatly influenced my approach to the field was a role model and source of inspiration to me, with his international outlook among many other things.

What job gave you the most useful training experience?

My first job at Annalise Dupont's Institute of Psychiatric Demography where I learned basic research methodology, team work, discipline and an international perspective.

Which book/ text has influenced you most?

The United Nations Universal Declaration of Human Rights. A large proportion of my professional and private life has been dedicated to the health consequences of human rights violations and how to prevent them.

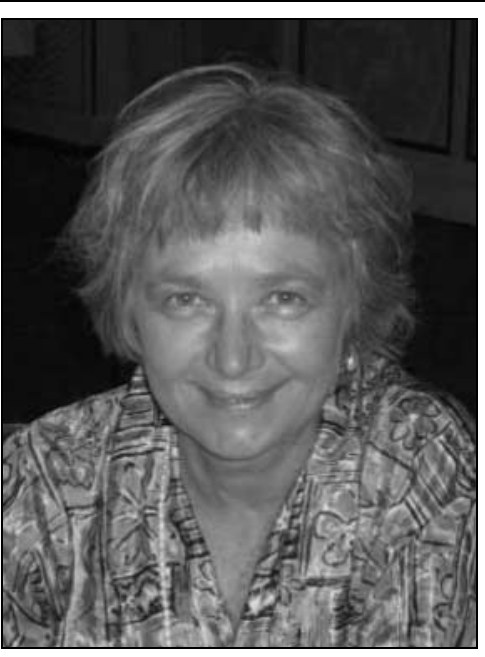

What research publication has had the greatest influence on your work? Education, Health and Behaviour by $\mathrm{M}$. Rutter, J. Tizard \& K. Whitmore (1970). This book was an inspiration during my first epidemiological research project and my later professional life.

What part of your work gives you the most satisfaction?

Working with international colleagues to reach consensus on common goals.

What part of your work do you least enjoy?

Dealing with staff conflicts.

What is the most promising opportunity facing the profession? Dealing with the effects of globalisation on mental health.

What is the greatest threat?

A shortage of psychiatrists and the risk posed to the profession by inadequate academic training.

What single change would substantially improve quality of care? Convincing politicians that allocating resources to mental health promotion and services is worthwhile. Allowing psychiatrists to concentrate on tasks appropriate to their expertise rather than routine tasks which can be carried out by others.

Do you think psychiatry is brainless or mindless?

believe that both should be recognised. There is a tendency to focus exclusively either on psychotherapeutic aspects or on neurobiological aspects. We also need a focus on the social dimension.

How would you entice more medical students into the profession?

Through an emphasis on psychiatry as a medical discipline and the opportunities to carry out research.

What is the most important advice you could offer to a new trainee? I would encourage them to be curious and open to the diversity of the field; to listen to patients' and relatives' stories without taking anything for granted; to look for a good mentor.

What are the main ethical problems that psychiatrists will face in the future?

There are several: increasing resources for those with mental illness; improving competence in dealing with patients from other cultures and maintaining professional standards.

How would you improve clinical psychiatric training?

Ensure pluralism in training programmes through inclusion of all relevant disciplines.

How should the role of the Royal College of Psychiatrists change? think that the College is doing great work, particularly with its international focus and attention to professional ethics.

What is the future for psychotherapy in psychiatry training and practice? I think a psychodynamic approach is important in establishing an alliance with patients and in understanding the ward environment. I believe that a cognitive approach is increasingly important in treatment.

What single area of psychiatric research should be given priority? Many areas should be given priority to counterbalance neurobiological and pharmacological research. These include epidemiological and health services research.

What single area of psychiatric practice is most in need of development?

As a consequence of the shortage of psychiatrists I think it is important to develop models of shared care with greater emphasis on a supervisory role for psychiatrists.

Dominic Fannon

doi: $10.1192 / p b . b p .107 .015545$ 